\title{
Diagnosing early onset dementia and then what? A frustrating system of aftercare resources
}

This article was published in the following Dove Press journal:

International Journal of General Medicine

18 January 2012

Number of times this article has been viewed

\author{
Z Chemali ${ }^{1-3}$ \\ S Schamber ${ }^{2}$ \\ EC Tarbi ${ }^{2}$ \\ D Acar ${ }^{1,2}$ \\ M Avila-Urizar ${ }^{2}$ \\ 'Harvard Medical School, \\ ${ }^{2}$ Departments of Neurology and \\ Psychiatry, Division of Cognitive \\ and Behavioral Neurology, Brigham \\ and Women's Hospital, ${ }^{3}$ Departments \\ of Psychiatry and Neurology, \\ Massachusetts General Hospital, \\ Boston, MA, USA
}

Correspondence: Z Chemali Harvard Medical School, Massachusetts General Hospital, Departments of Psychiatry and Neurology, 55 Fruit Street, Boston, MA 02। I4, USA

Tel: + I 6177245600

Fax: +| $617726754 \mid$

Email zelchemali@partners.org
Abstract: Recent studies indicate that the prevalence of early onset dementia (EOD) is more common than it was once presumed. As such, and considering the substantial challenges EOD presents to the patient, caregivers, and health care providers, this study sought to investigate the mechanism of care delivered to these patients. A medical record chart review was conducted for 85 patients attending a memory disorder unit who initially presented to rule out EOD as a working diagnosis. The results suggest that while the majority of these patients received an extensive work-up and were heavily medicated, they remained at home, where they lacked adequate age-related services and could not be placed, despite the crippling caregiver burden. This manuscript is a platform to discuss our current system limitations in the care of these patients with an eye on new opportunities for this challenging group.

Keywords: early onset dementia, social work, services, caregiving

\section{Introduction}

Although the prevalence of early onset dementia (EOD) (dementia in individuals $<65$ years old) was once assumed to be very low, additional data have shown that prevalence has varied between 15.1-43.9/100,000 for early onset Alzheimer's disease (AD), ${ }^{1}$ while the prevalence of frontotemporal dementia (FTD) has varied from 2.7/100,000 in the Netherlands ${ }^{2}$ to $15.1 / 100,000$ in Cambridge, UK. ${ }^{3}$ In Japan, a new study published in Stroke showed that the overall prevalence of EOD was 42.3/100,000 - divided into vascular dementia (42.6\%), AD (25.6\%), traumatic brain injury $(7.1 \%)$, dementia with Lewy bodies, and Parkinson's disease $(6.2 \%)$, FTD (2.6\%) and other (16\%). ${ }^{4}$ In Europe, according to an AD Europe 2009 study, all dementia prevalence was 38-420/100,000 with AD being 15.1-153/100,000 and FTD being 4.0-15.4/100,000. ${ }^{5}$

However uncommon, EOD poses a real problem to the patient, their family members and caregivers, doctors, health services, and residential programs. ${ }^{6}$ The challenges faced include difficulty in making the diagnosis, the impact of the diagnosis on family members and children (as EOD patients may still have younger offspring), employment, family and personal finances, and quality of life. In this pilot study, we seek to identify the work-up and interventions sought in the process of diagnosis, shedding light on the limited help offered after a diagnosis is made under the current system. We end by putting forth possible solutions to the challenges encountered in treating and caring for these individuals at both an individualized and system level of care. 


\section{Methods}

\section{Participants and experimental procedures}

We conducted a medical record chart review, after internal review board review and approval, for patients presenting to a memory disorder unit in Boston, Massachusetts, from 2005 to 2008. Patients' charts were flagged if the presenting complaint was to rule out a dementia diagnosis and if they were $<65$ years old. Dementia was defined as a group of symptoms in more than one domain affecting intellectual and social functions severely enough to interfere with daily functioning. ${ }^{7}$ This resulted in the study of the charts of 85 patients (mean age [standard deviation]: 55.19 [7.51] years). Table 1 illustrates the demographic composition of the group.

We define social work intervention to include full psychosocial assessment including family and individual patient history, current strengths, and supports and assessment of risk factors. Social workers are part of the interdisciplinary team within the clinic and therefore are accessible to all patients and family members, either at their own request or at the request/referral from a provider. It should be noted that social work intervention was obtained at the discretion of the physician; the incidence of social work intervention did not necessarily reflect issues related to the patient (as difficult family dynamics, for instance, often prompt intervention) or disease severity. Separate appointments are scheduled for the patient and the family. They have the option of keeping the appointment and deciding on the extent of social work involvement. The social work visits for both patients and their families were counted in the study.

Analyses centered on the relationship between social work intervention and several outcome measures. The outcome measures included the type of work-up the patient received (magnetic resonance imaging [MRI], positron emission tomography, single positron emission computed tomography, neuropsychological evaluation, or any combination of these), the type of medications the patient was given, any changes in patient location/treatment over time, or any newly developing medical complaints.

\section{Analysis}

Data were analyzed with PASW Statistics (v 18.0; IBM Corp, Armonk, NY), using an alpha level of 0.05 for significance. The relationship between categorical variables of interest was investigated using Pearson's chi-square analyses; the distribution of variables of interest within the sample was assessed using chi-square goodness of fit tests. To examine trends in the Mini-Mental Status Exam (MMSE) data which was used to measure dementia severity, univariate analysis of variance
Table I Patient characteristics

\begin{tabular}{|c|c|c|}
\hline Characteristics & $\mathbf{N}$ & Frequency \\
\hline \multicolumn{3}{|l|}{ Gender } \\
\hline Male & 35 & $41.20 \%$ \\
\hline Female & 50 & $50.80 \%$ \\
\hline \multicolumn{3}{|l|}{ SW Consult } \\
\hline No & 48 & $56.50 \%$ \\
\hline Yes & 37 & $43.50 \%$ \\
\hline \multicolumn{3}{|l|}{ Home at diagnosis } \\
\hline No & 3 & $3.50 \%$ \\
\hline Yes & 82 & $96.50 \%$ \\
\hline \multicolumn{3}{|l|}{ Last recorded location } \\
\hline Home & 77 & $90.60 \%$ \\
\hline Hospice & I & $1.20 \%$ \\
\hline Nursing home & 6 & $7.10 \%$ \\
\hline Unknown & I & $1.20 \%$ \\
\hline \multicolumn{3}{|l|}{ Diagnosis } \\
\hline ETOH & 2 & $2.40 \%$ \\
\hline HIV & I & $1.20 \%$ \\
\hline MS & 10 & $11.80 \%$ \\
\hline Neoplasm & I & $1.20 \%$ \\
\hline Neurodegenerative & 50 & $58.80 \%$ \\
\hline$A D$ & 19 & \\
\hline FTD & 13 & \\
\hline $\mathrm{MCl}$ & 9 & \\
\hline MIX & 3 & \\
\hline PCA & I & \\
\hline PD & 5 & \\
\hline Other & 2 & $2.40 \%$ \\
\hline CNOS/Behcet & I & \\
\hline $\mathrm{NPH}$ & I & \\
\hline $\mathrm{TBI}$ & 10 & $11.80 \%$ \\
\hline$A D D+M D D$ & 9 & $10.60 \%$ \\
\hline ADD & 6 & \\
\hline MDD & 3 & \\
\hline First MMSE, mean (SD) & 60 & $27.25(3.34)$ \\
\hline MMSE follow-up (I-2 years), mean (SD) & 20 & $25.45(4.5 \mathrm{I})$ \\
\hline MMSE follow-up (nursing home), mean (SD) & 3 & $25.67(1.16)$ \\
\hline
\end{tabular}

Notes: Details the patient characteristics in terms of demographic breakdown, as well as in terms of the outcome variables. *Other, HIV, ETOH, CNOS/Behcet, Mix, Neoplasm, NPH, PCA.

Abbreviations: SW, social work; ETOH, alcoholism; HIV, human immunodeficiency virus; MS, multiple sclerosis; $A D$, Alzheimer's disease; FTD, frontotemporal dementia; $\mathrm{MCl}$, mild cognitive impairment; MIX, mixed dementia; PCA, posterior cerebral artery stroke; PD, Parkinson's disease; NPH, normal pressure hydrocephalus; TBI, traumatic brain injury; ADD, attention deficit disorder; MDD, major depressive disorder; MMSE, Mini-Mental State Examination; SD, standard deviation.

was utilized. Analyses investigating the impact of social work intervention on the outcome variables excluded patients whose final diagnosis was cognitive decline due to severe executive dysfunction as a result of attention deficit disorder or major depressive disorder. Patients with either of these disorders were included in the initial data sampling procedure as they presented to the clinic with EOD symptoms but were excluded from analyses when their final diagnosis was linked to longstanding single-domain cognitive and/or emotional difficulty. Seventy-six patients were included in the final analysis. 


\section{Results}

On average, patients were seen 2.3 times/year by a clinic provider. The disorders requiring the most visits until a final diagnosis was made were early onset dementia - Alzheimer type and FTD. Looking closely at the neurodegenerative disorders as a unique group resulted in the data presented in Table 2, concerning specific patient work-up.

Donepezil was the most used monotherapy. It should be noted that the majority of the individuals within the study were on one or two medications $\left(\chi^{2}[4, \mathrm{~N}=76]=24.13, P<0.001\right)$. Additionally, as dementia severity advanced as assessed by the MMSE, so did polypharmacy $(\mathrm{F}[4,48]=6.17, P<0.001$; 0 meds: $\mathrm{MMSE}=28.00[1.46]$ versus four medications: MMSE $=21.00$ [1.31] [mean (standard deviation)]). Interestingly, there was no difference in MMSE score between the groups receiving or not receiving social work intervention. Neuropsychological testing was the most used diagnostic modality (alone or in combination) regardless of patients' diagnoses. MRI was the first imaging modality used. Patients would move on to have positron emission tomography when brain MRI was negative for specific findings but clinical suspicion was high for a neurodegenerative disease.

Although social work intervention was rated as the most meaningful supportive intervention, it did not significantly correlate with any of the outcome measures (type or number of medications, type or number of patient work-ups, and reason for medical visit). Social work intervention did not limit polypharmacy or the number of diagnostic procedures patients undertook until a diagnosis was made. Additionally, social work intervention did not correlate with the patient's location at diagnosis nor their last recorded location. The out-of-home placement is generally considered for patients with severe agitation and/or severe incontinence. The authors note again that this decision is often family centered and closely related to access to those facilities. Given this, in this study, it was not surprising to find that the majority of the group was at home at diagnosis $\left(\chi^{2}[1, \mathrm{~N}=76]=64.47, P<0.001\right)$ and at last follow-up $\left(\chi^{2}[3, \mathrm{~N}=76]=169.37, P<0.001\right)$. In fact, only five patients experienced a change in their treatment setting over the course of the 3 years that they were followed. Of those five individuals, three had a social work intervention, while two did not.

\section{Discussion}

EOD poses a significant diagnostic challenge as it frequently presents in a young adult population burdened by a wide range of behavioral, cognitive and psychiatric symptoms. Predominant psychiatric presentation without significant cognitive deficits or atypical presentation of a common dementia challenges the family and the health care provider, often leading to diagnosis delay. ${ }^{8-11}$ In addition, when a diagnosis is made, while this brings "relief" for the already frustrated caregiver, it launches the much more tasking phase of ensuring that adequate care is provided for the patient. Beyond these challenges - already advanced in a previously published body of work ${ }^{6}$ - the stigma and taboo of cognitive illness and "losing one's mind" - especially a young person's mind do not help in advancing the situation. Instead, they often interfere with appropriate communication within the family, creating additional hurdles for the care of the patient. ${ }^{12,13}$

This study shows that the issues for patients and their families move quickly from diagnostic to psychosocial. ${ }^{13,14}$ Social work consultation is integrated into care as early dementia affects patients who are still at an active age and vibrant in their social circles; the financial, health, wellbeing, social structure, and family losses create chaos in the family; wreak havoc in marital dynamics; and bring major uncertainties in terms of unemployment, financial issues, and long-term health care. ${ }^{13,15}$ Given these concerns, a social work intervention is often sought, although it is unclear if this changes the course and/ or the delivery of care. The cost of social work consultation

Table 2 Patient work-up subcategory diagnosis

\begin{tabular}{|c|c|c|c|c|c|c|}
\hline Diagnosis & $\begin{array}{l}\text { Patients, } \\
\text { N }\end{array}$ & $\begin{array}{l}\text { SW } \\
\text { intervention }\end{array}$ & $\begin{array}{l}\geq 1 \\
\text { med }\end{array}$ & $\begin{array}{l}\geq 1 \\
\text { work-up }\end{array}$ & $\begin{array}{l}\text { Home at } \\
\text { diagnosis }\end{array}$ & $\begin{array}{l}\text { Home } \\
\text { now }\end{array}$ \\
\hline$A D$ & 19 & 5 & 5 & 4 & 5 & 5 \\
\hline FTD & 13 & 7 & 6 & 7 & 7 & 6 \\
\hline PD & 5 & 3 & 3 & 3 & 3 & 3 \\
\hline $\mathrm{MCl}$ & 9 & 3 & 3 & 2 & 3 & 3 \\
\hline TBI & 10 & 8 & 8 & 8 & 8 & 7 \\
\hline MS & 10 & 6 & 4 & 5 & 6 & 5 \\
\hline Other* & 10 & I & 10 & 10 & 10 & 10 \\
\hline
\end{tabular}

Notes: Illustrates more completely the work-up each patient received, organized by neurodegenerative diagnosis (major subgroups). *Includes neoplasm, human immunodeficiency virus, alcohol-related dementia, normal pressure hydrocephalus, etc.

Abbreviations: SW, social work; AD, Alzheimer's disease; FTD, frontotemporal dementia; PD, Parkinson's disease; TBI, traumatic brain injury; MS, multiple sclerosis; MCI, mild cognitive impairment. 
is usually covered by health insurance and is billable under the patients' mental health benefit. Worth noting is the family's preference for keeping patients at home or placing them in a facility. The dearth of services becomes evident when families and providers seek appropriate care.

Physicians are quickly confronted with system issues as soon as the diagnosis is confirmed. Assessment tools specifically targeting this young population are not generally readily available and those that are fail to portray and quantify the severity of the deficit in executive functions and instrumental activities of daily living. ${ }^{6,12,14-16}$ In fact, clinicians are often confronted with a young adult who, although having a somewhat preserved MMSE score, performs poorly when it comes to executive functioning (such as planning, organization, good judgment, etc). The answers to those unbearable changes is often medication, which is quickly escalated to poly-pharmacy, with little effect on behavior. This puts the patient at higher risk from side effects without alleviating the disease symptomatology. In fact, these deficits are the hallmark of how a patient's life will fair. Moreover, with no solutions in sight, the cognitive impairment coupled with the behavioral changes are felt as a burden to the whole family and impact heavily on the public health sector, which is unable to meet the demands and needs of the young population. Clinicians have to adjust quickly to a list of complaints presented by family members and they address this knowing they cannot do much to alleviate this suffering. Family members are "left in the dark" in attending to a high level of stress and depression due to a crippling burden of care. Although caregiver's burnout is often recognized by clinicians, few know how to deal with its consequences and, if they do know, they find themselves unable to offer much as community services are a rare commodity. ${ }^{17,18}$ The authors recognize the importance of social worker intervention to help caregivers deal with stress and ultimately avoid "burnout."

In addition, stigma against those with dementia remains high and numerous psychosocial issues exist for patients and families affected by EOD. The emotional challenge of adjusting to a difficult and debilitating diagnosis affecting the life course of the patient and their family is horrendous. ${ }^{19}$ Families are alone as they face unique caregiver challenges. ${ }^{13,20,21}$

As the young EOD patient is often forced to stop working, the patient's partner or child, often identified as the main caregiver, must face the challenge of full- or part-time employment in addition to overseeing the patient's care and other household responsibilities. The caregiver often files for family leave or ends up doing odd jobs at odd hours to be able to care for their loved ones at home while maintaining some form of income. ${ }^{19}$ As shown in this study, the dearth of services for patients with EOD, coupled with a lack in coverage of community services, leads to the majority of individuals remaining at home for many years after their diagnosis is established. The resource access challenge is evidenced on the home front as well. ${ }^{16,22}$ Home-based services remain inaccessible to many - especially those without considerable financial resources. Many rehabilitative therapies are not mainstreamed or reimbursed by insurance companies. Additionally, families also face this challenge when looking for suitable nursing home placement. As shown in Figure 1, the state of Massachusetts still lacks a comprehensive holding environment, public programs, and services for EOD patients. Currently, the authors know of no facilities dedicated to providing care to patients with EOD. They may be placed in nursing homes or hospitals that cannot meet all their needs as young patients with a different course or manifestation of illness. They receive some care, but it is not optimal to their needs or to the needs of their families when it comes to specific athletic, recreational, or occupational activities. Because of their younger age, their spouse and children may have different needs than the family members of older patients. To date, there are few programs that include training for facility staff and family members, although no one refutes that those specialized training centers would provide the best care to patients with EOD in the long run.

Given the issues enumerated, this study - despite its small scale - found that the current state of affairs should not continue as such. We were fortunate to capture cases of EOD with different etiologies. Of course, one could extrapolate, for example, on the number of underdiagnosed EOD cases due to alcoholism that create problems in the public health system with its current available resources and scarce finances. This study has shown that, regardless of the etiology of the dementia, there is a lack of treatment and resources.

\section{Study limitations}

The study offers the limitations of a retrospective pilot study in describing the findings in a limited group of patients already self-selected as they were attending a memory disorder unit. The results should be assessed with this in mind and should not be generalized before longitudinal studies are undertaken. In addition, this study may have failed to consider available services if they were not visible to the studied community, although all known state agencies, EOD support groups, and social work resources were enlisted before observations were stated. 


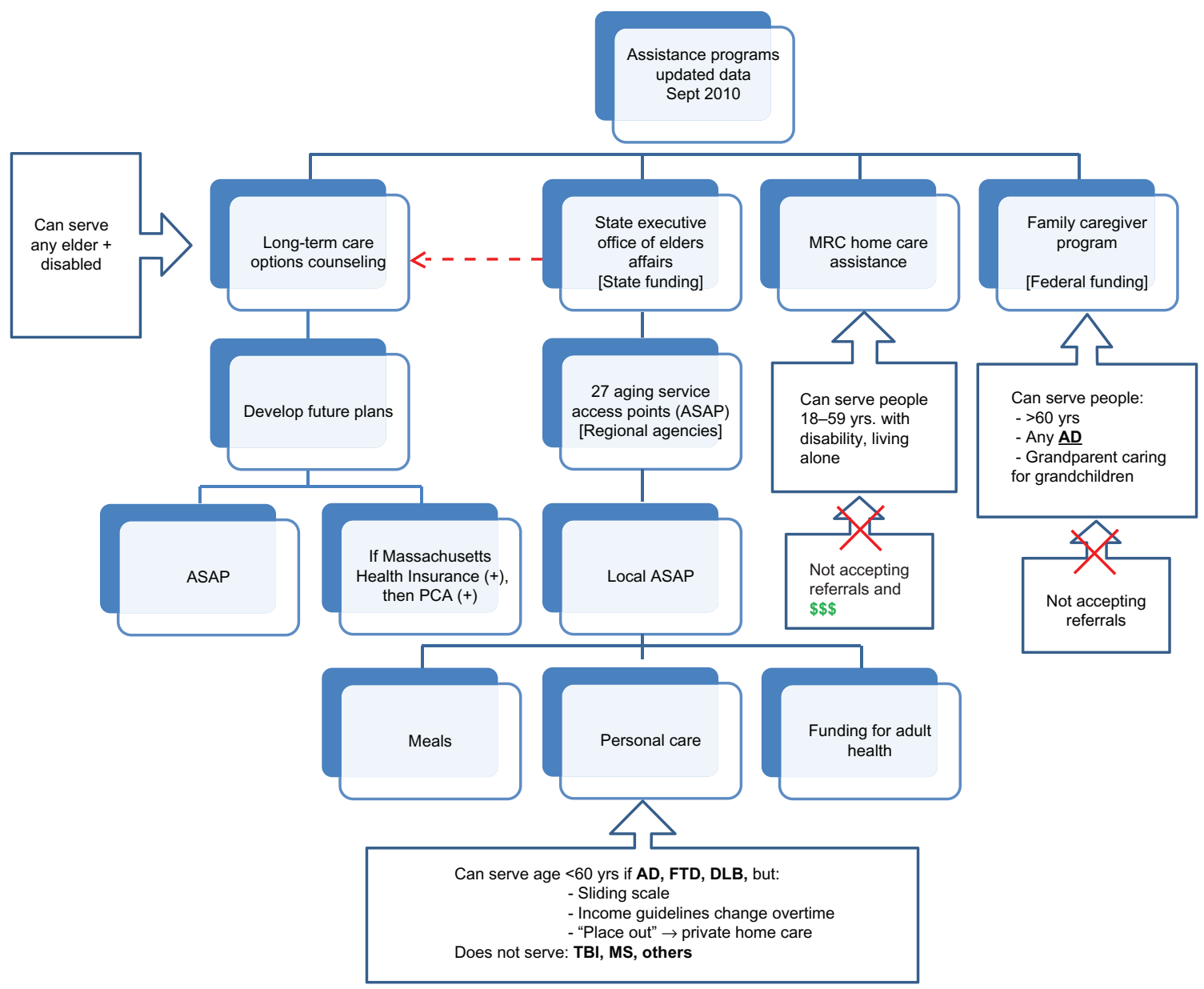

Figure I Nursing home flow chart.

Notes: Outlines the state services and funding options available for home care assistance programs in Massachusetts.

Abbreviations: MRC, Massachusetts Rehabilitation Commission; PCA, private care assistant; AD, Alzheimer's disease; FTD, frontotemporal dementia; DLB, dementia with Lewy bodies; TBI, traumatic brain injury; MS, multiple sclerosis.

\section{Conclusion}

Dementia causes considerable suffering for patients and their families. EOD has an even higher burden. Extensive population studies on the incidence and prevalence of EOD are for the most part still lacking. ${ }^{23}$ For this and many other reasons, the practical matching of resources to EOD patients still lags. The interventions to date, including social work, are not sufficient to tackle the problem when there is a lack of resources and absence of policy targeting the rights and needs of a younger population. While the authors acknowledge the limitations of this retrospective pilot study, they consider that the data collected and presented on the EOD patient population are invaluable. They encourage future research to validate the results in longitudinal studies and rely on a strong legislature. Future recommendations include education; increased awareness placed on the needs of this younger population suffering from dementia; and flexibility in giving space to potential emerging roles to be performed in nursing, social work, and hospice and palliative care coupled with statewide initiatives.

Establishing a diagnosis is not enough. It is not an endgoal either. With this in mind, the authors would like to open the floor to a serious discussion among caregivers, patients, legislatures, insurance companies, and places of residence that will not shy away from tackling the tough issues and the formulation of a sustainable working plan for caring for patients with EOD.

\section{Disclosure}

The authors report no conflicts of interest in this work.

\section{References}

1. Harvey RJ, Skelton-Robinson M, Rosser MN. The prevalence and causes of dementia in people under the age of 65 years. J Neurol Neurosurg Psychiatry. 2003;74(9):1206-1209.

2. Rosso SM, Donker Kaat L, Baks T, et al. Frontotemporal dementia in The Netherlands: Patient characteristics and prevalence estimates from a population-based study. Brain. 2003;126(Pt 9):2016-2022. 
3. Mercy L, Hodges JR, Dawson K, Barker RA, Brayne C. Incidence of early-onset dementias in Cambridgeshire, United Kingdom. Neurology. 2008;71(19):1496-1499.

4. Ikejima C, Yasuno F, Mizukami K, Sasaki M, Tanimukai S, Asada T. Prevalence and causes of early-onset dementia in Japan: A populationbased study. Stroke. 2009;40(8):2709-2714.

5. Alzheimer Europe. Prevalence of early-onset dementia in Europe [web page on the Internet]. Luxembourg: Alzheimer Europe; 2011. Available from: http://www.alzheimer-europe.org/index.php/DE/Research/ European-Collaboration-on-Dementia/Prevalence-of-dementia/ Prevalence-of-early-onset-dementia-in-Europe. Accessed November 26, 2011.

6. Chemali Z, Withall A, Daffner KR. The plight of caring for young patients with frontotemporal dementia. Am J Alzheimers Dis Other Demen. 2010;25(2):109-115.

7. American Psychiatric Association. Diagnostic and Statistical Manual of Mental Disorders. 4th ed. 1994.

8. Folstein MF, Folstein SE, McHugh PR. "Mini-mental state." A practical method for grading the cognitive state of patients for the clinician. J Psychiatr Res. 1975:12 (3):189-198.

9. Woodley JD, Khan BK, Murthy NK, Miller BL, Rankin RP. The diagnostic challenge of psychiatric symptoms in neurodegenerative disease: rate and risk factors for prior diagnosis in patients with early onset dementia. J Clin Psychiatry. 2011;72(2):126-133.

10. Thomas-Anterion C, Laurent B. Diagnostic Precoce de la Maladie d'Alzeimer [Early diagnosis of Alzheimer's disease]. Rev Prat. 1998; 48(17):1889-1890. French.

11. Mendez MF. The accurate diagnosis of early onset dementia. Int J Psych Med. 2006;36(4):401-412.

12. Podell K, Torres K. Affective symptoms in early onset dementia. Neurol Clin. 2011;29(1):99-114.

13. Sampson EL, Warren JD, Rossor MN. Young Onset Dementia. Postgrad Med J. 2004;80(941):125-139.
14. Arai A, Matsumoto T, Ikeda M, Arai Y. Do family caregivers perceive more difficulty when they look after patients with early onset dementia compared to those with late onset dementia? Int J Geriatr Psychiatry. 2007;22(12):1255-1261.

15. Werner P, Stein-Shrachman I, Korczyn AD. Early onset dementia: clinical and social aspects. Int Psychogeriatr. 2009;21(4):631-636.

16. Van Vliet D, Bakker C, Koopmans RT, Vernooij-Dassen MJ, Verhey FR, de Vugt ME. Research protocol of the NeedYD-study (Needs in Young onset Dementia): a prospective cohort study on the needs and course of early onset dementia. BMC Geriatr. 2010;10:13.

17. Workman B, Dickson F, Green S. Early dementia-Optimal management in general practice. Aust Fam Physician. 2010;39(10):722-726.

18. Kaiser S, Panegyres PK. The psychosocial impact of young onset dementia on spouses. Am J Alzheimers Dis Other Demen. 2007;21: 398-402.

19. Silverstein NM, Wong CM, Bucek KE. Adult day health care for participants with Alzheimer's disease. Am J Alz Dis Other Dement. 2010; 25(3):276-283.

20. Adams KB. The transition to caregiving: the experience of family members embarking on the dementia caregiving career. J Gerontol Soc Work. 2006;47(3-4):3-29.

21. Gelman CR, Greer C. Young children in early onset Alzheimer's disease families: Research gaps and emerging service needs. Am JAlz Dis Other Demen. 2011;26(1):29-35.

22. Van Vliet D, de Vugt ME, Bakker C, Koopmans RT, Verhey FR. Impact of early onset dementia on caregivers: a review. Int J Geriatr Psychiatry. 2010;25(11):1091-1100.

23. Gauthier SG. Alzheimer disease: The benefit of early treatment. Eur $J$ Neurol. 2005;12 Suppl(3):11-16.

24. Garre-Olmo J, Genis Batlle D, del Mar Fernandez M, et al. Incidence and subtypes of early-onset dementia in geographically defined general population. Neurology. 2010;75(14):1249-1255.
International Journal of General Medicine

\section{Publish your work in this journal}

The International Journal of General Medicine is an international, peer-reviewed open-access journal that focuses on general and internal medicine, pathogenesis, epidemiology, diagnosis, monitoring and treatment protocols. The journal is characterized by the rapid reporting of reviews, original research and clinical studies across all disease areas.

\section{Dovepress}

A key focus is the elucidation of disease processes and management protocols resulting in improved outcomes for the patient.The manuscript management system is completely online and includes a very quick and fair peer-review system. Visit http://www.dovepress.com/ testimonials.php to read real quotes from published authors. 